\title{
Comparison of an 8-Channel and a 32-Channel Coil for High-Resolution fMRI at $7 \mathrm{~T}$
}

\author{
Roy Salomon · Jana Darulova $\cdot$ Mayur Narsude • \\ Wietske van der Zwaag
}

Received: 17 May 2013/Accepted: 28 May 2013/Published online: 8 June 2013

(C) Springer Science+Business Media New York 2013

\begin{abstract}
Multi-channel receive array rf-coils have become widely available for fMRI. The improved SNR and possibility of acquisition acceleration through parallel imaging are especially beneficial for high-resolution studies. In this study, an 8-channel and a 32-channel coil were compared in a high-resolution finger tapping fMRI experiment at $7 \mathrm{~T}$. $1.3 \mathrm{~mm}^{3}$ resolution data acquired with the 32-channel coil provided higher image- and temporal SNR and yielded higher BOLD sensitivity measures, notably higher cluster sizes in MI/SI and increased z-scores, though not an increase in percent signal change. For sub-millimeter resolution fMRI data acquired with the 32-channel coil smaller clusters were found, though percent signal changes were significantly larger, due to reduced partial volume effects. These results demonstrate the utility of the use of an array coil with a large number of receive elements for high-resolution fMRI at ultra-high field.
\end{abstract}

Electronic supplementary material The online version of this article (doi:10.1007/s10548-013-0298-6) contains supplementary material, which is available to authorized users.

R. Salomon · J. Darulova

Laboratory of Cognitive Neuroscience (LNCO), Ecole

Polytechnique Fédérale de Lausanne (EPFL), Lausanne,

Switzerland

R. Salomon

Center for Neuroprosthetics, Ecole Polytechnique Fédérale de

Lausanne (EPFL), Lausanne, Switzerland

J. Darulova $\cdot$ M. Narsude $\cdot$ W. van der Zwaag $(\bowtie)$

Laboratory for Functional and Metabolic Imaging (LIFMET),

CIBM-AIT, Ecole Polytechnique Fédérale de Lausanne (EPFL),

Station 6, 1015 Lausanne, Switzerland

e-mail: wietske.vanderzwaag@epfl.ch
Keywords fMRI $\cdot$ Coil comparison $\cdot 7 \mathrm{~T}$. BOLD sensitivity $\cdot$ Sensory-motor cortex

\section{Introduction}

The use of multi-channel rf-coils in MRI has two major advantages: (1) the signal-to-noise ratio (SNR) levels are generally higher than with single-channel coils (Mekle et al. 2008) and (2) the different spatial characteristics of the individual coil loops allow acceleration of the image acquisition through parallel imaging (Wiggins et al. 2006). Receive arrays with a large number of coils generally present more signal inhomogeneity, with higher SNR values in cortical regions and smaller signal increases in central brain regions (Kaza et al. 2011; Mekle et al. 2008). The SNR increase provided by a 32-channel coil has been shown to improve BOLD sensitivity in cortical regions in low-resolution fMRI at 3T (Kaza et al. 2011).

At ultra-high field, physiological noise becomes more prominent; larger cardiac and respiratory induced signal fluctuations reduce the maximum attainable temporal SNR (tSNR) (Bianciardi et al. 2009). tSNR is of importance to fMRI experiments, because the features of interest are activation-induced temporal signal fluctuations. Thus, increased image SNR at ultra-high field is often traded for spatial resolution, where the long echo trains associated with large imaging matrices are reduced via parallel imaging techniques to keep TE approximately equal to $\mathrm{T}_{2}^{*}$. Partial volume effects and resulting false-positive BOLD signal detection are reduced at higher spatial resolution (van der Zwaag et al. 2009) and in high-resolution data, thermal noise is dominant (Bodurka et al. 2007), further motivating the use of rf-arrays with a high number of receive elements. Thus, use of a 32-channel coil is 
expected to be especially advantageous for high-resolution fMRI.

Here, we compare the tSNR values and BOLD sensitivity measures in a simple high-resolution motor-task fMRI experiment at $7 \mathrm{~T}$. Two rf-coils were compared: an 8-channel cylindrical transmit/receive coil and a 32-channel dome-shaped receive array combined with a single channel transmit coil.

\section{Methods}

Twelve healthy volunteers were scanned in the $7 \mathrm{~T}$ (Siemens, Germany) after providing written informed consent. Six volunteers ( 2 female) were scanned with the 8-channel coil (Rapid Biomedical, Germany) and the remaining six (3 female) with the 32-channel coil (Nova Medical, MA, USA). All volunteers performed the same simple visually cued finger tapping task for each 12 min run. For details, see supplementary methods. EPI data were acquired either with a voxel size of $1.3 \mathrm{~mm}^{3}$ isotropic (standard) or with a voxel size of $0.75 \times 0.75 \times 1.0 \mathrm{~mm}^{3}$ (high resolution) High resolution data were only acquired with the 32-channel coil as the 8-channel coil did not support the GRAPPA $=4$ acceleration required to keep the echo-trainlength down. TR and spatial coverage were kept constant across runs. Standard fMRI data processing was carried out using FSL, with and without spatial smoothing. For further acquisition and data processing details, as well as g-factor maps, please refer to the supplementary methods.

Measures of BOLD sensitivity were: the size of the largest cluster in the sensory-motor cortex (MI/SI); the percent signal change in the 1000 most active voxels and the z-score of the 1000th most active voxel. The z-score of the 1000th ranked voxel was chosen because maximum z-scores were infinite in several subjects. Values were compared between groups of data with an unpaired $t$ test.
Temporal SNR was measured as a voxel's mean time course value divided by the standard deviation in time. Values were averaged over a parietal white matter ROI away from any areas of activation. Image SNR values were taken from the same ROI, but obtained by subtracting consecutive volumes of the fMRI train and dividing the mean signal with the standard deviation of the absolute of the noise over the ROI following (Lai and Glover 1998).

\section{Results and Discussion}

Use of the 32-channel coil resulted both with and without smoothing in significantly higher image SNR and temporal SNR values than use of the 8-channel coil for the brain region scanned in these experiments (Table 1). BOLD sensitivity, as measured by the number of voxels in the largest activated cluster in MI/SI was significantly higher for the 32-channel coil ( $p<0.05$, unpaired $t$ test, both smoothed and unsmoothed) and the 1000th highest z-score value was also significantly higher $(p<0.01$, both smoothed and unsmoothed). The percentage signal change measured in the 1000 most active voxels was not significantly different, showing that the measured signal between the two groups was the same, yet contained less noise in the case of the 32-channel coil.

In contrast, when the higher signal of the 32-channel coil was traded for higher spatial resolution and a voxel size of $0.56 \mu \mathrm{l}$ used instead of $2.2 \mu \mathrm{l}$, image SNR and tSNR were both reduced compared to the standard voxel, unsmoothed 8-channel coil data. While there was no significant difference in number of detected voxels or z-score of the 1000th most active voxel, the percent signal change in the 1000 most active voxels was significantly larger for the high resolution data than for the standard resolution data $(p<0.05$, both coils, both for smoothed and unsmoothed). The reduced partial volume effects in the

Table 1 SNR and BOLD sensitivity measures for the simple motor task using different rf-coils

\begin{tabular}{|c|c|c|c|c|c|c|}
\hline & $\begin{array}{l}\text { 8-Channel coil, } \\
2.2 \mu \text { l voxel } \\
\text { smoothed }\end{array}$ & $\begin{array}{l}\text { 32-Channel coil, } \\
2.2 \mu 1 \text { voxel } \\
\text { smoothed }\end{array}$ & $\begin{array}{l}\text { 8-Channel coil, } \\
2.2 \mu \text { l voxel } \\
\text { unsmoothed }\end{array}$ & $\begin{array}{l}\text { 32-Channel coil, } \\
2.2 \mu \text { l voxel } \\
\text { unsmoothed }\end{array}$ & $\begin{array}{l}\text { 32-Channel coil, } \\
0.6 \mu \text { l voxel } \\
\text { unsmoothed }\end{array}$ & $\begin{array}{l}\text { 32-Channel coil, } \\
0.6 \mu 1 \text { voxel } \\
3096 \text { voxels }\end{array}$ \\
\hline Image SNR & $52 \pm 2$ & $145 \pm 2 *$ & $21 \pm 1$ & $66 \pm 1^{*}$ & $9.8 \pm 0.3$ & \\
\hline Temporal SNR & $44 \pm 1$ & $131 \pm 2 *$ & $18 \pm 1$ & $57 \pm 1^{*}$ & $8.7 \pm 0.2$ & \\
\hline \#Active voxels in MI/SI & $1,490 \pm 160$ & $6,440 \pm 550^{*}$ & $620 \pm 80$ & $4,095 \pm 460 *$ & $1,192 \pm 240$ & \\
\hline Size MI/SI cluster $\left(\mathrm{cm}^{3}\right)$ & $3.3 \pm 0.4$ & $14.1 \pm 1.2 *$ & $1.4 \pm 0.2$ & $9.0 \pm 1.0 *$ & $0.7 \pm 0.1$ & \\
\hline Signal change $(\%)$ & $2.9 \pm 0.1$ & $3.5 \pm 0.1$ & $4.28 \pm 0.16$ & $4.76 \pm 0.2$ & $7.5 \pm 0.3$ & $5.4 \pm 0.2$ \\
\hline Threshold & $4.1 \pm 0.1$ & $8.1 \pm 0.3^{*}$ & $3.2 \pm 0.1$ & $5.89 \pm 0.2 *$ & $3.8 \pm 0.1$ & $2.9 \pm 0.1$ \\
\hline
\end{tabular}

All values are given as the mean \pm SE of the mean over subjects. Percent signal change was measured in the 1000 most active voxels. Threshold is the z-score of the 1000th most active voxel, or the threshold z-score above which the percent signal change was measured

*Indicates a significant difference with the 8 -channel coil value ( $p<0.05$, unpaired $t$ test). For the high resolution data, percent signal change and threshold values were also obtained for the top 3906 ranked voxels 
$8 \mathrm{Ch}$.
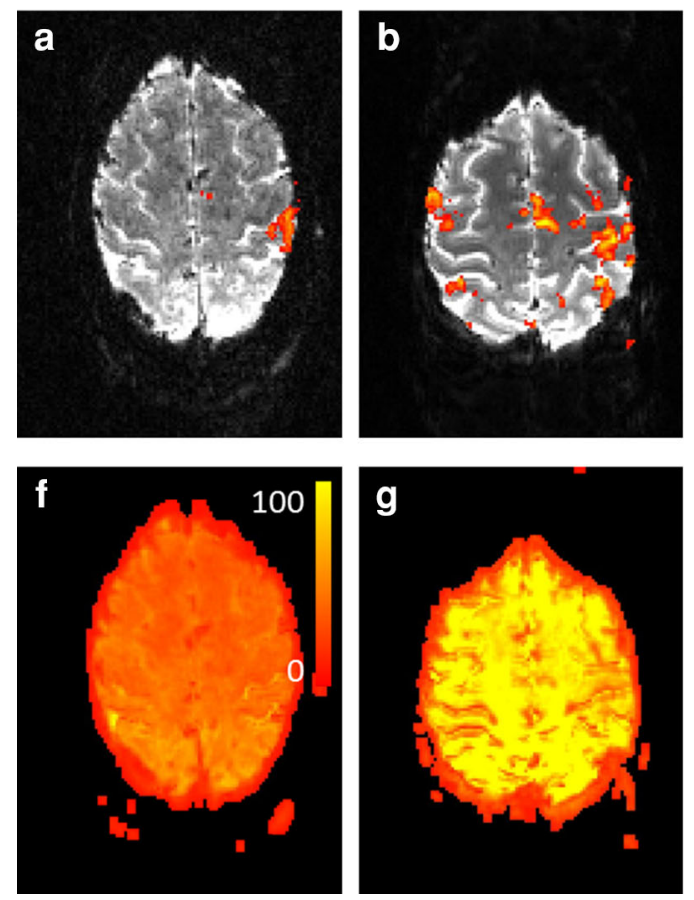

$8 \mathrm{Ch}$.
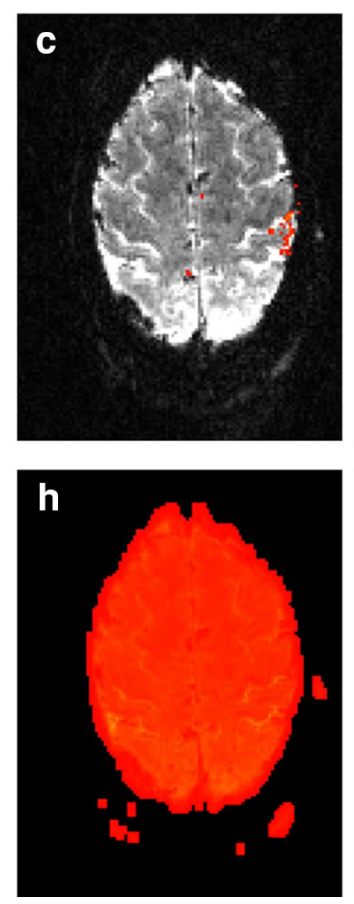

$32 \mathrm{Ch}$.
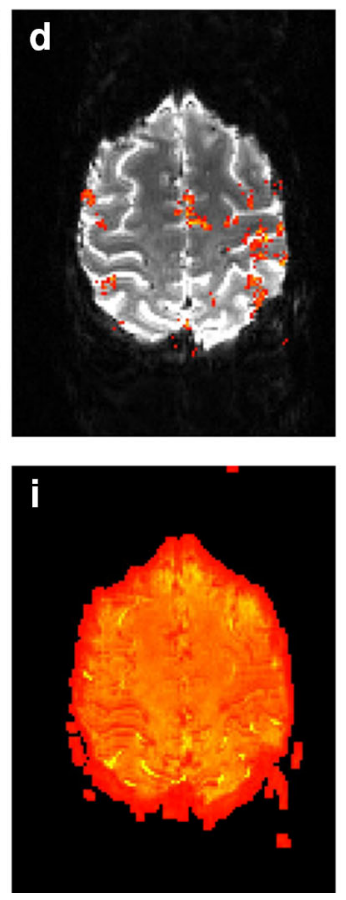

$32 \mathrm{Ch}$.
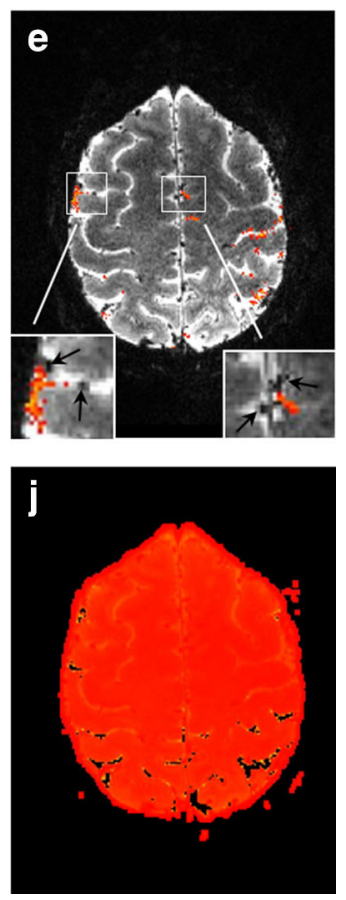

Fig. 1 a-e Activation maps of representative subjects. a Activation map acquired with the 8 -channel coil, spatial resolution $1.3 \mathrm{~mm}^{3}$, $2 \mathrm{~mm}$ smoothing. b Activation map acquired with the 32-channel coil, spatial resolution $1.3 \mathrm{~mm}^{3}, 2 \mathrm{~mm}$ smoothing. c 8 -channel coil, $1.3 \mathrm{~mm}^{3}$, no smoothing. d 32-channel coil, $1.3 \mathrm{~mm}^{3}$, no smoothing. e Activation map acquired with the 32-channel coil, spatial resolution $0.75 \times 0.75 \times 1.0 \mathrm{~mm}^{3}$, no smoothing. Black arrows in the insets indicate the position of veins. All activation maps are scaled from $\mathrm{z}=2.3$ to 10 and are shown overlaid on an exemplar of the echoplanar image train they were generated from. Note the extended task-

high resolution data increased the number of high percentage change voxels as more active voxels fell completely within the grey matter, while the increased noise reduced the z-score threshold to a value compared to that of the 8-channel coil data. Even when the number of voxels was increased to 3906 , to match the volume of voxels covered by the $10001.3 \mathrm{~mm}^{3}$ voxels, the percentage change remained higher than that in the standard resolution datasets acquired with either coil, while the threshold was significantly below that of the 8-channel coil, further emphasizing the importance of partial volume effects in the $1.3 \mathrm{~mm}^{3}$ data.

Activation maps (Fig. 1a-e) and tSNR maps (Fig. 1f-j) of representative subjects show the same trends. For equal voxel sizes, activation is more widespread in the 32-channel coil data compared to the 8 channel coil (Fig. 1a-d). When the spatial resolution is increased (Fig. 1e), activation is clearly limited to the grey matter ribbon in the sensory-motor cortex and supplementary motor area. Note that active voxels are not found in the expected location of draining veins (inserts, Fig. 1e), activated regions in the 32 channel coil data $(\mathbf{f}-\mathbf{j})$ identical slices of tSNR maps of representative subjects. $\mathbf{f}$ tSNR map acquired with the 8-channel coil, spatial resolution $1.3 \mathrm{~mm}^{3}, 2 \mathrm{~mm}$ smoothing. G: tSNR map acquired with the 32-channel coil, spatial resolution $1.3 \mathrm{~mm}^{3}$, $2 \mathrm{~mm}$ smoothing. h 8-channel coil, $1.3 \mathrm{~mm}^{3}$, no smoothing. i 8channel coil, $1.3 \mathrm{~mm}^{3}$, no smoothing. $\mathbf{j}$ tSNR map acquired with the 32-channel coil, spatial resolution $0.75 \times 0.75 \times 1.0 \mathrm{~mm}^{3}$. All tSNR maps are scaled from 0 to 100 . Note the homogeneity of the tSNR maps in general and higher values for the 32-channel coil

probably because of the short local $\mathrm{T}_{2}^{*}$. Temporal SNR values were homogeneous throughout the imaged slices (Fig. 1f-j) and highest for the smoothed data sets. In the unsmoothed data, standard voxel size data acquired with the 32-channel coil showed highest tSNR values, and the high resolution data the lowest, but tSNR was still sufficient to detect BOLD signal in the expected locations.

\section{Conclusion}

Use of a 32-channel coil data for rf-reception provides significantly increased temporal SNR values in $7 \mathrm{~T}$ fMRI that can be traded for higher spatial resolution of the fMRI data.

Acknowledgments This work was supported by the Centre d'Imagerie BioMédicale (CIBM) of the UNIL, UNIGE, HUG, CHUV, EPFL and the Leenaards and Jeantet Foundations. R.S. was supported by the National Center of Competence in Research (NCCR) "SYNAPSY - The Synaptic Bases of Mental Diseases" financed by the Swiss National Science Foundation (No 51AU40_125759). 


\section{References}

Bianciardi M, van Gelderen P, Duyn JH, Fukunaga M, de Zwart JA (2009) Making the most of fMRI at $7 \mathrm{~T}$ by suppressing spontaneous signal fluctuations. NeuroImage 44(2):448-454

Bodurka J, Ye F, Petridou N, Murphy K, Bandettini PA (2007) Mapping the MRI voxel volume in which thermal noise matches physiological noise-implications for fMRI. NeuroImage 34(2):542-549

Kaza E, Klose U, Lotze M (2011) Comparison of a 32-channel with a 12-channel head coil: are there relevant improvements for functional imaging? J Magn Reson Imaging 34(1):173-183

Lai S, Glover GH (1998) Three-dimensional spiral fMRI technique: a comparison with 2D spiral acquisition. Magn Reson Med 39(1):68-78
Mekle R, van der Zwaag W, Joosten A, Gruetter R (2008) Comparison of three commercially available radio frequency coils for human brain imaging at $3 \mathrm{~T}$. Magma 21(1-2):53-61

van der Zwaag W, Marques JP, Hergt M, Gruetter R (2009) Investigation of high-resolution functional magnetic resonance imaging by means of surface and array radiofrequency coils at 7 T. Magn Reson Imaging 27(8):1011-1018

Wiggins GC, Triantafyllou C, Potthast A, Reykowski A, Nittka M, Wald LL (2006) 32-channel $3 \mathrm{~T}$ receive-only phased-array head coil with soccer-ball element geometry. Magn Reson Med 56(1):216-223 